\title{
Evaporator for orc cycle with recirculating heat carring water - computational model
}

Tomasz KUJAWA, Władysław NOWAK

DOI: 10.30464/jmee.2018.2.4. 301

Cite this article as:

Kujawa T., Nowak W. Evaporator for orc cycle with recirculating heat carring water - computational model. Journal of Mechanical and Energy Engineering, Vol. 2(42), No. 4, 2018, pp. 301-310.

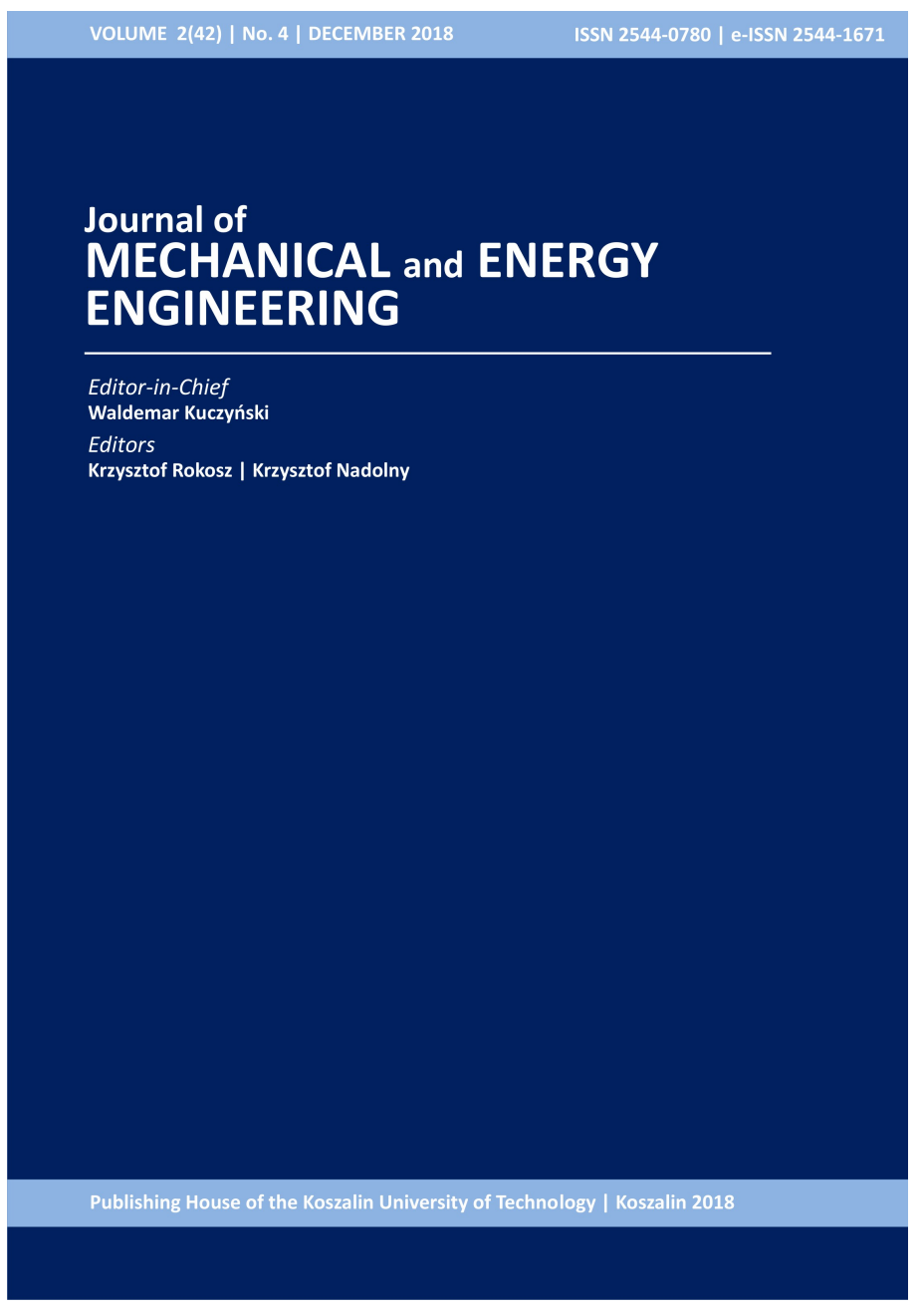

Journal of Mechanical and Energy Engineering

Website: jmee.tu.koszalin.pl

ISSN (Print): 2544-0780

ISSN (Online): 2544-1671

Volume: 2(42)

Number: 4

Year: 2018

Pages: $301-310$

Article Info:

Received 26 October 2018

Accepted 4 December 2018

\section{Open Access}

This article is distributed under the terms of the Creative Commons Attribution 4.0 (CC BY 4.0) International License (http://creativecommons.org/licenses/by/4.0/), which permits unrestricted use, distribution, and reproduction in any medium, provided you give appropriate credit to the original author(s) and the source, provide a link to the Creative Commons license, and indicate if changes were made. 


\title{
EVAPORATOR FOR ORC CYCLE WITH RECIRCULATING HEAT CARRING WATER - COMPUTATIONAL MODEL
}

\author{
Tomasz KUJAWA ${ }^{1 *}$, Władysław NOWAK ${ }^{1}$ \\ ${ }^{1 *}$ West Pomeranian University of Technology, Szczecin, Faculty of Mechanical Engineering \\ and Mechatronics, Department of Heat Engineering, al. Piastów 7, 70-310 Szczecin, Poland, \\ e-mail: tomasz.kujawa@ zut.edu.pl
}

(Received 26 October 2018, Accepted 4 December 2018)

\begin{abstract}
The following paper presents an ORC installation including an evaporator with recirculation (heat carrying water exiting the evaporator is redirected to its inlet). It covers the calculations of inlet/outlet temperature of the evaporator taking into account a variant recirculation coefficient. Formulas for heat transfer between heat carrying water and working fluid inside evaporator are also included in this paper. The calculations are based on properly defined average specific heat. The analysis shows that the system performance depends on heat carrying water inlet temperature, on heat carrying water flow rate and the recirculation coefficient.
\end{abstract}

Keywords: evaporator, recirculation, average specific heat, ORC power plant, mathematical modeling

\section{INTRODUCTION}

This paper presents a computational model for a power plant with single subcritical ORC loop (Fig. 1) [10-12] that instead of a classic evaporator includes one with recirculating heat carrying water. The computational model is based on a general steady state energy balance

$$
\dot{E}_{d}=\dot{E}_{w} .
$$

In order to obtain a simple computational model, two types of average specific heat were used: $\left.c\right|_{0} ^{T}$ and $\left.c\right|_{T_{2}} ^{T_{1}}$. The first average specific heat $\left.c\right|_{0} ^{T}$ was used to determine the specific enthalpy of heat carrying water

$$
h=\left.c\right|_{0} ^{T} T
$$

that allows to calculate the enthalpy of water flux using the following formula:

$$
\dot{H}=\dot{m} \cdot h \text {. }
$$

A relation between two average specific heats $\left.c\right|_{0} ^{T_{1}}$ and $\left.c\right|_{0} ^{T_{2}}$ was used to derive $\left.c\right|_{T_{2}} ^{T_{1}}$.

The computational model [12] is based on an assumptions that the energy is supplied to the system by hot water with mass flow rate $\dot{m}_{s}$ and temperature $T_{s 1}$.

The main purpose of the formulas within the computational model is to estimate the evaporator inlet and outlet temperatures $T_{s 1}^{z}$ and $T_{s 2}^{z}$ taking into account temperature $T_{s 1}$, recirculation coefficient $z$ and evaporator temperature difference

$$
\Delta T_{s}^{z}==T_{s 1}^{z}-T_{s 2}^{z} .
$$

The analysis conducted proves that the evaporator inlet and outlet temperatures can be calculated using the following formulas:

$$
T_{s 1}^{z}=f\left(T_{s 1}, z, \Delta T_{s}^{z}\right) \text { and } T_{s 2}^{z}=f\left(T_{s 1}, z, \Delta T_{s}^{z}\right) .
$$

The analysis also shows that the heat $\dot{Q}_{s}$ transferred from water to working fluid, basing on the 
energy balance, can be calculated according to the following formula:

$$
\dot{Q}_{s}=(1+z) \dot{m}_{s} \bar{c}_{s}\left(T_{s 1}^{z}-T_{s 2}^{z}\right)==\dot{m}_{n} \Delta h_{p a r}
$$

The mass flow rate of heat carrying water $\dot{m}_{s 1}$ suppling the counter current working fluid pre-heater can be calculated using energy balance of this heat exchanger and average specific heat $\bar{c}_{s 2}$.

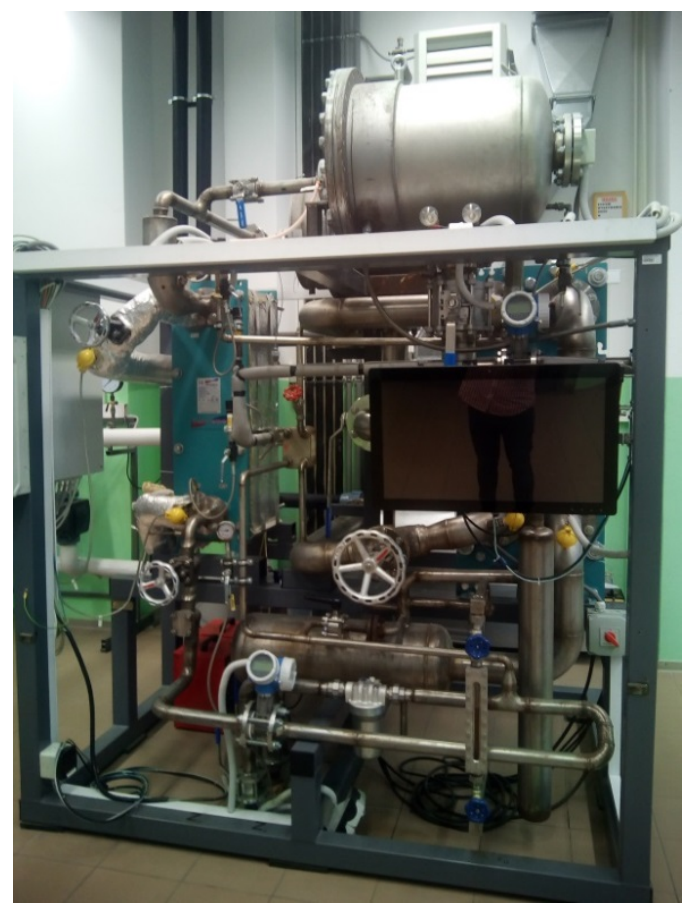

Fig. 1. ORC power plant supplied with water at $100^{\circ} \mathrm{C}$ (Department of Heat Engineering, West Pomeranian University of Technology in Szczecin, operating factor $\mathrm{R} 227 \mathrm{ea}, 9 \mathrm{~kW}_{\text {el }}$ power) [11]

\section{ORC POWER PLANT WITH RECIR- CULATING HEAT CARRYING WATER}

In a power plant with single ORC loop it is possible to select a proper working fluid. Currently, there is a variety of substances that can serve as working fluid: natural pure substances (butane, izobutane, propane), synthetic pure substances and blends [1-8, 14-16]. Utilization of organic substance allows to achieve better working conditions comparing to the water (for the same pressure and temperature). The main advantage of those substances over the water is low evaporation temperature (for some of them even below $100^{\circ} \mathrm{C}$ ).

A scheme of a single loop ORC power plant with recirculating heat carrying water evaporator is presented in Fig. 2.

The principle of operation for ORC power plant with recirculating heat carrying water evaporator is simple. First, the heat carrying water is directed to the evaporator. Due to recirculation, flow rate through evaporator (node A) is a sum of circulating water $\dot{m}_{s}$ and the recirculating water $\Delta \dot{m}_{s}$. Thus, the temperature at evaporator inlet drops to $T_{s 1}^{z}$. The flow rate at the evaporator outlet $\left(\dot{m}_{s}+\Delta \dot{m}_{s}\right)$ splits into two streams (node B). The $\Delta \dot{m}_{s}$ stream is redirected through by-pass to the evaporator inlet where it meets heat carrying water, and the $\dot{m}_{s}$ stream goes further into the system. Then the $\dot{m}_{s}$ stream splits into two streams $\dot{m}_{s 1}$ and $\dot{m}_{s 2}:$ the $\dot{m}_{s 1}$ stream is directed to ORC working fluid pre-heater $\left(\dot{m}_{s 1}\right.$ can be determined using energy balance equation for the preheater); the $\dot{m}_{s 2}$ stream can be calculated according to the formula $\dot{m}_{s 2}=\dot{m}_{s}-\dot{m}_{s 1}$, at temperature $T_{s 2}^{z}$ it reconnects the $\dot{m}_{s 1}$ stream at temperature $T_{s 3}$ at node C. The mixed stream with flow rate $\dot{m}_{s}=\dot{m}_{s 1}+\dot{m}_{s 1}$ and temperature $T_{s 4}$ is directed to a heat exchanger where the temperature rises from $T_{s 4}$ to $T_{s 1}$. On the secondary side of the heat exchanger there is a waste heat carrier with flow rate $\dot{m}_{o}$ that operates in a temperature range between $T_{o 1}$ and $T_{o 2}$.

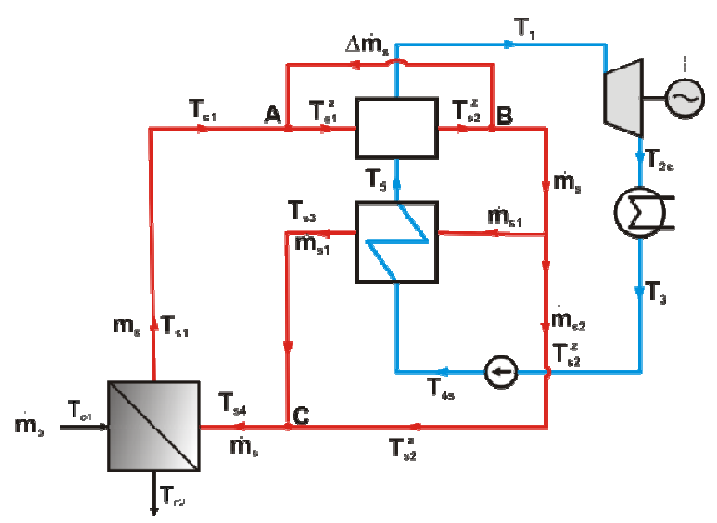

Fig. 2. A scheme of single loop ORC power plant with recirculating heat carrying water evaporator [12]

\section{A METHOD FOR MODELLING OF RECIRCULATING HEAT CARRYING WATER EVAPORATOR}

Fig. 3 shows a scheme of recirculating heat carrying water evaporator. It includes three balance sections (space inside evaporator occupied by liquid $\mathbf{a}$ and space occupied $\mathbf{c}$ occupied by evaporating working fluid) together with all important parameters necessary for computation.

The energy balance equation for water space inside the evaporator, including the heat flux $\dot{Q}_{s}$ 
supplied to the evaporating medium (balance section a in Fig. 3) looks as follow:

$$
\left.(1+z) \dot{m}_{s} \cdot c\right|_{0} ^{T_{s 1}^{z}} T_{s 1}^{z}=\left.(1+z) \dot{m}_{s} \cdot c\right|_{0} ^{T_{s 2}^{z}} T_{s 2}^{z}+\dot{Q}_{s}
$$

or after rearrangement:

$$
\dot{Q}_{s}=(1+z) \dot{m}_{s}\left[\left.c\right|_{0} ^{T_{s 1}^{z}} T_{s 1}^{z}-\left.c\right|_{0} ^{T_{s 2}^{z}} T_{s 2}^{z}\right] .
$$

Next, the average specific heat is introduced $\bar{c}_{s}=\left.c\right|_{T_{s 2}^{z}} ^{T_{s 1}^{z}}$.

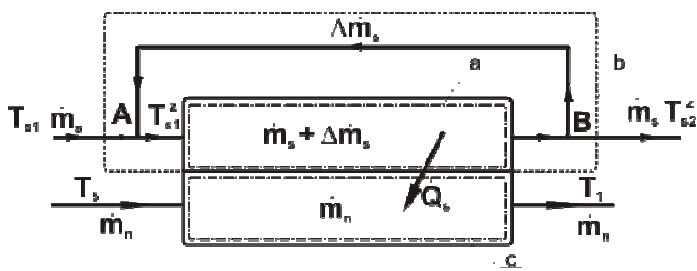

Fig. 3. Scheme of recirculating heat carrying water evaporator [12]

This heat is defined as a function of two temperatures of recirculating water at evaporator inlet and outlet, $T_{s 1}^{z}$ and $T_{s 2}^{z}$, respectively, and two average specific heats $\left.c\right|_{0} ^{T_{s 1}^{z}}$ and $\left.c\right|_{0} ^{T_{s 2}^{z}}$ :

$$
\bar{c}_{s}=\left.c\right|_{T_{s 2}^{z}} ^{T_{s 1}^{z}}=\frac{\left.c\right|_{0} ^{T_{s 1}^{z}} T_{1}^{z}-\left.c\right|_{0} ^{T_{s 2}^{z}} T_{s 2}^{z}}{T_{s 1}^{z}-T_{s 2}^{z}} .
$$

After substituting (1a) into (2):

$$
\bar{c}_{s}\left(T_{s 1}^{z}-T_{s 2}^{z}\right)=\left.c\right|_{0} ^{T_{s 1}^{z}} T_{s 1}^{z}-\left.c\right|_{0} ^{T_{s 2}^{z}} T_{s 2}^{z},
$$

after simple rearrangements, the heat flux from water to evaporating working medium can be written in following form:

$$
\dot{Q}_{s}=(1+z) \dot{m}_{s} \cdot \bar{c}_{s}\left(T_{s 1}^{z}-T_{s 2}^{z}\right) .
$$

In order to determine the recirculating water temperature, the energy balance equation for node $\mathrm{A}$ is used:

$$
\left.\dot{m}_{s} \cdot c\right|_{0} ^{T} T_{s 1} T_{s 1}+\left.z \dot{m}_{s} \cdot c\right|_{0} ^{T_{s 2}^{z}} T_{s 2}^{z}=\left.(1+z) \dot{m}_{s} \cdot c\right|_{0} ^{T_{s 1}^{z}} T_{s 1}^{z}
$$

or after rearrangements the formula (4) takes the following form:

$$
\left.c\right|_{0} ^{T_{s 1}} T_{s 1}-\left.c\right|_{0} ^{T_{s 1}^{z}} T_{s 1}^{z}=z\left(\left.c\right|_{0} ^{T_{s 1}^{z}} T_{s 1}^{z}-\left.c\right|_{0} ^{T_{s 2}^{z}} T_{s 2}^{z}\right) .
$$

After introducing another average specific heat $\bar{c}_{s 1}=\left.c\right|_{T_{s 1}^{z}} ^{T_{s 1}^{1}}$ defined as follow:

$$
\bar{c}_{s 1}=\left.c\right|_{T_{s 1}^{z}} ^{T_{s 1}}=\frac{\left.c\right|_{0} ^{T_{s 1}} T_{s 1}-\left.c\right|_{0} ^{T_{s 1}^{z}} T_{s 1}^{z}}{T_{s 1}-T_{s 1}^{z}}
$$

and average specific heat $\bar{c}_{s}$ from (2a) into (4a), it can be rewritten to the following form:

$$
\bar{c}_{s 1}\left(T_{s 1}-T_{s 1}^{z}\right)=z \cdot \bar{c}_{s}\left(T_{s 1}^{z}-T_{s 2}^{z}\right) .
$$

After proper rearrangements, the temperature or recirculating water can be calculated according to the following formula:

$$
T_{s 1}^{z}=T_{s 1}-z \cdot \frac{\bar{c}_{s}}{\bar{c}_{s 1}} \Delta T_{s}^{z}
$$

To derive the formula for water temperature at evaporator outlet, it is necessary to make an energy balance for section $\mathbf{b}$ (Fig. 3):

$$
\left.\dot{m}_{s} \cdot c\right|_{0} ^{T_{s 1}} T_{s 1}=\dot{Q}_{s}+\left.\dot{m}_{s} \cdot c\right|_{0} ^{T_{s 2}} T_{s 2},
$$

that after substituting (3) takes the following form:

$$
\left.c\right|_{0} ^{T_{s 1}} T_{s 1}-\left.c\right|_{0} ^{T_{s 2}} T_{s 2}=(1+z) \bar{c}_{s}\left[T_{s 1}^{z}-T_{s 2}^{z}\right] .
$$

After using the definition of average specific heat $\bar{c}_{s 2}=\left.c\right|_{T_{s 2}^{z}} ^{T_{s 1}}:$

$$
\bar{c}_{s 2}=\left.c_{s 2}\right|_{T_{s 1}^{z}} ^{T_{s 1}}=\frac{\left.c\right|_{0} ^{T_{s 1}} T_{s 1}-\left.c\right|_{0} ^{T_{s 2}^{z}} T_{s 2}^{z}}{T_{s 1}-T_{s 2}^{z}}
$$

the (7a) can be transformed into a formula for water temperature $T_{s 2}^{z}$ :

$$
\bar{c}_{s 2}\left(T_{s 1}-T_{s 2}^{z}\right)=(1+z) \bar{c}_{s}\left(T_{s 1}^{z}-T_{s 2}^{z}\right)
$$

or:

$$
T_{s 2}^{z}=T_{s 1}-(1+z) \cdot \frac{\bar{c}_{s}}{\bar{c}_{s 2}} \Delta T_{s}^{z}
$$

Using the formulas (6) and (9a) that allow to calculate water temperature at evaporator inlet and outlet, $T_{s 1}^{z}$ and $T_{s 2}^{z}$, respectively, it is possible to evaluate the temperature difference $T_{s 2}^{z}$ :

$$
T_{s 1}^{z}-T_{s 2}^{z}=\beta \Delta T_{s}^{z},
$$

where:

$$
\beta=\frac{\bar{c}_{s}}{\bar{c}_{s 2}}+z\left(\frac{\bar{c}_{s}}{\bar{c}_{s 2}}-\frac{\bar{c}_{s}}{\bar{c}_{s 1}}\right) \text {. }
$$

The numerical values of average specific heat $\bar{c}_{s}, \bar{c}_{s 1}$ and $\bar{c}_{s 2}$ were calculated using specific enthalpy $h^{\prime}$ for saturated water available in REFPROP 9 software [9]: 


$$
\begin{gathered}
\bar{c}_{s}=\frac{h^{\prime}\left(T_{s 1}^{z}\right)-h^{\prime}\left(T_{s 2}^{z}\right)}{T_{s 1}^{z}-T_{s 2}^{z}}= \\
=\frac{h^{\prime}\left(T_{s 1}-z \Delta T_{s}^{z}\right)-h^{\prime}\left(T_{s 1}-(1+z) \Delta T_{s}^{z}\right]}{\Delta T_{s}^{z}}, \\
\bar{c}_{s 1}=\frac{h^{\prime}\left(T_{s 1}\right)-h^{\prime}\left(T_{s 1}^{z}\right)}{T_{s 1}-T_{s 1}^{z}}=\frac{h^{\prime}\left(T_{s 1}\right)-h^{\prime}\left(T_{s 1}-z \Delta T_{s}^{z}\right)}{z \Delta T_{s}^{z}}, \\
\text { for } z \neq 0, \\
\bar{c}_{s 2}=\frac{h^{\prime}\left(T_{s 1}\right)-h^{\prime}\left(T_{s 2}^{z}\right)}{T_{s 1}-T_{s 2}^{z}}=\frac{h^{\prime}\left(T_{s 1}\right)-h^{\prime}\left(T_{s 1}-(1+z) \Delta T_{s}^{z}\right]}{(1+z) \Delta T_{s}^{z}}
\end{gathered}
$$

The results of average specific heat calculations are presented in tables 1-6. They served as a base for average specific heat charts $\bar{c}_{s}=f\left(T_{s 1}, z\right), \bar{c}_{s 1}=$ $=f\left(T_{s 1}, z\right), \quad \bar{c}_{s 2}=f\left(T_{s 1}, z\right)$ (Fig. 4-6) and ratios of average specific heat chart $\bar{c}_{s} / \bar{c}_{s 1}=f\left(T_{s 1}, z\right)$, $\bar{c}_{s} / \bar{c}_{s 2}=f\left(T_{s 1}, z\right)$ and $\beta=f\left(T_{s 1}, z\right)-$ Fig. 7-9.

The analysis conducted, for the water temperature range $T_{s 1}=220 \div 90^{\circ} \mathrm{C}$ and recirculation coefficient range $z=0 \div 12$, shows that $\bar{c}_{s} / \bar{c}_{s 1}=f\left(T_{s 1}, z\right) \cong 1$; $\bar{c}_{s} / \bar{c}_{s 2}=f\left(T_{s 1}, z\right) \cong 1$ and $\beta=f\left(T_{s 1}, z\right) \cong 1$.
Taking the above into consideration, basing on formula (6), the temperature of recirculating water at the evaporator inlet can be calculated according to

$$
T_{s 1}^{z}=T_{s 1}-z \cdot \Delta T_{s}^{z} .
$$

Taking the above into consideration, basing on formula (6), the temperature of recirculating water at

\begin{tabular}{|c|c|c|c|c|c|c|c|c|c|c|c|c|c|c|}
\hline \multirow[b]{2}{*}{$\mathrm{Z}$} & \multicolumn{14}{|c|}{ Specific heat at constant pressure $[\mathrm{kJ} /(\mathrm{kgK})]$ for temperatures $\left[{ }^{\circ} \mathrm{C}\right]$ : } \\
\hline & 220 & 210 & 200 & 190 & 180 & 170 & 160 & 150 & 140 & 130 & 120 & 110 & 100 & 90 \\
\hline 0 & 4.595 & 4.536 & 4.484 & 4.438 & 4.397 & 4.361 & 4.329 & 4.302 & 4.278 & 4.257 & 4.239 & 4.225 & 4.213 & 4.203 \\
\hline 1 & 4.536 & 4.484 & 4.438 & 4.397 & 4.361 & 4.329 & 4.302 & 4.278 & 4.257 & 4.239 & 4.225 & 4.213 & 4.203 & 4.194 \\
\hline 2 & 4.484 & 4.438 & 4.397 & 4.361 & 4.329 & 4.302 & 4.278 & 4.257 & 4.239 & 4.225 & 4.213 & 4.203 & 4.194 & 4.189 \\
\hline 3 & 4.438 & 4.397 & 4.361 & 4.329 & 4.302 & 4.278 & 4.257 & 4.239 & 4.225 & 4.213 & 4.203 & 4.194 & 4.189 & \\
\hline 4 & 4.397 & 4.361 & 4.329 & 4.302 & 4.278 & 4.257 & 4.239 & 4.225 & 4.213 & 4.203 & 4.194 & 4.189 & & \\
\hline 5 & 4.361 & 4.329 & 4.302 & 4.278 & 4.257 & 4.239 & 4.225 & 4.213 & 4.203 & 4.194 & 4.189 & & & \\
\hline 6 & 4.329 & 4.302 & 4.278 & 4.257 & 4.239 & 4.225 & 4.213 & 4.203 & 4.194 & 4.189 & & & & \\
\hline 7 & 4.302 & 4.278 & 4.257 & 4.239 & 4.225 & 4.213 & 4.203 & 4.194 & 4.189 & & & & & \\
\hline 8 & 4.278 & 4.257 & 4.239 & 4.225 & 4.213 & 4.203 & 4.194 & 4.189 & & & & & & \\
\hline 9 & 4.257 & 4.239 & 4.225 & 4.213 & 4.203 & 4.194 & 4.189 & & & & & & & \\
\hline 10 & 4.239 & 4.225 & 4.213 & 4.203 & 4.194 & 4.189 & & & & & & & & \\
\hline 11 & 4.225 & 4.213 & 4.203 & 4.194 & 4.189 & & & & & & & & & \\
\hline 12 & 4.213 & 4.203 & 4.194 & 4.189 & & & & & & & & & & \\
\hline
\end{tabular}
the evaporator inlet can be calculated according to

$$
T_{s 1}^{z}=T_{s 1}-z \cdot \Delta T_{s}^{z} .
$$

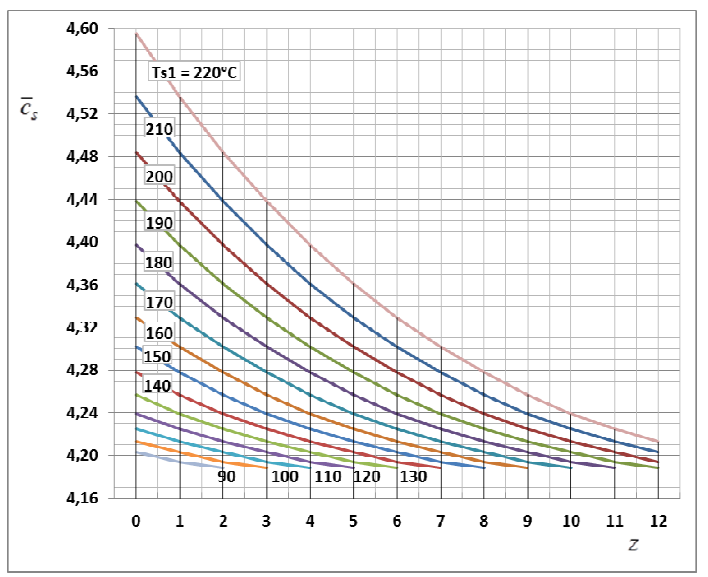

Fig. 4.Average specific heat $\bar{c}_{s}=f\left(T_{s 1}, z\right)$

Tab. 1. Average specific heat $\bar{c}_{s}=f\left(T_{s 1}, z\right)$ 
Tab. 2. Average specific heat $\bar{c}_{s 1}=f\left(T_{s 1}, z\right)$

\begin{tabular}{|c|c|c|c|c|c|c|c|c|c|c|c|c|c|c|}
\hline \multirow[b]{2}{*}{ Z } & \multicolumn{14}{|c|}{ Specific heat at constant pressure $[\mathrm{kJ} /(\mathrm{kgK})]$ for temperatures $\left[{ }^{\circ} \mathrm{C}\right]$ : } \\
\hline & 220 & 210 & 200 & 190 & 180 & 170 & 160 & 150 & 140 & 130 & 120 & 110 & 100 & 90 \\
\hline 1 & 4.595 & 4.536 & 4.484 & 4.438 & 4.397 & 4.361 & 4.329 & 4.302 & 4.278 & 4.257 & 4.239 & 4.225 & 4.213 & 4.203 \\
\hline 2 & 4.566 & 4.510 & 4.461 & 4.418 & 4.379 & 4.345 & 4.316 & 4.290 & 4.268 & 4.248 & 4.232 & 4.219 & 4.208 & 4.199 \\
\hline 3 & 4.538 & 4.486 & 4.440 & 4.399 & 4.362 & 4.331 & 4.303 & 4.279 & 4.258 & 4.240 & 4.226 & 4.214 & 4.203 & 4.195 \\
\hline 4 & 4.513 & 4.464 & 4.420 & 4.381 & 4.347 & 4.318 & 4.292 & 4.269 & 4.250 & 4.234 & 4.220 & 4.209 & 4.200 & 4.193 \\
\hline 5 & 4.490 & 4.443 & 4.402 & 4.365 & 4.333 & 4.305 & 4.281 & 4.260 & 4.242 & 4.227 & 4.215 & 4.205 & 4.197 & \\
\hline 6 & 4.469 & 4.424 & 4.385 & 4.351 & 4.321 & 4.294 & 4.272 & 4.252 & 4.236 & 4.222 & 4.211 & 4.201 & & \\
\hline 7 & 4.449 & 4.407 & 4.370 & 4.337 & 4.309 & 4.284 & 4.263 & 4.245 & 4.230 & 4.217 & 4.207 & & & \\
\hline 8 & 4.430 & 4.391 & 4.356 & 4.325 & 4.299 & 4.276 & 4.256 & 4.239 & 4.225 & 4.213 & & & & \\
\hline 9 & 4.413 & 4.376 & 4.343 & 4.314 & 4.289 & 4.267 & 4.249 & 4.233 & 4.220 & & & & & \\
\hline 10 & 4.398 & 4.362 & 4.331 & 4.304 & 4.280 & 4.260 & 4.243 & 4.228 & & & & & & \\
\hline 11 & 4.383 & 4.350 & 4.320 & 4.295 & 4.273 & 4.254 & 4.238 & & & & & & & \\
\hline 12 & 4.370 & 4.338 & 4.311 & 4.286 & 4.266 & 4.248 & & & & & & & & \\
\hline
\end{tabular}

Tab. 3.Average specific heat $\bar{c}_{s 2}=f\left(T_{s 1}, z\right)$

\begin{tabular}{|c|c|c|c|c|c|c|c|c|c|c|c|c|c|c|}
\hline \multirow[b]{2}{*}{$\mathrm{Z}$} & \multicolumn{14}{|c|}{ Specific heat at constant pressure $[\mathrm{kJ} /(\mathrm{kgK})]$ for temperatures $\left[{ }^{\circ} \mathrm{C}\right]$ : } \\
\hline & 220 & 210 & 200 & 190 & 180 & 170 & 160 & 150 & 140 & 130 & 120 & 110 & 100 & 90 \\
\hline 0 & 4.595 & 4.536 & 4.484 & 4.438 & 4.397 & 4.361 & 4.329 & 4.302 & 4.278 & 4.257 & 4.239 & 4.225 & 4.213 & 4.203 \\
\hline 1 & 4.566 & 4.510 & 4.461 & 4.418 & 4.379 & 4.345 & 4.316 & 4.290 & 4.268 & 4.248 & 4.232 & 4.219 & 4.208 & 4.199 \\
\hline 2 & 4.538 & 4.486 & 4.440 & 4.399 & 4.362 & 4.331 & 4.303 & 4.279 & 4.258 & 4.240 & 4.226 & 4.214 & 4.203 & 4.195 \\
\hline 3 & 4.513 & 4.464 & 4.420 & 4.381 & 4.347 & 4.318 & 4.292 & 4.269 & 4.250 & 4.234 & 4.220 & 4.209 & 4.200 & 4.193 \\
\hline 4 & 4.490 & 4.443 & 4.402 & 4.365 & 4.333 & 4.305 & 4.281 & 4.260 & 4.242 & 4.227 & 4.215 & 4.205 & 4.197 & \\
\hline 5 & 4.469 & 4.424 & 4.385 & 4.351 & 4.321 & 4.294 & 4.272 & 4.252 & 4.236 & 4.222 & 4.211 & 4.201 & & \\
\hline 6 & 4.449 & 4.407 & 4.370 & 4.337 & 4.309 & 4.284 & 4.263 & 4.245 & 4.230 & 4.217 & 4.207 & & & \\
\hline 7 & 4.430 & 4.391 & 4.356 & 4.325 & 4.299 & 4.276 & 4.256 & 4.239 & 4.225 & 4.213 & & & & \\
\hline 8 & 4.413 & 4.376 & 4.343 & 4.314 & 4.289 & 4.267 & 4.249 & 4.233 & 4.220 & & & & & \\
\hline 9 & 4.398 & 4.362 & 4.331 & 4.304 & 4.280 & 4.260 & 4.243 & 4.228 & & & & & & \\
\hline 10 & 4.383 & 4.350 & 4.320 & 4.295 & 4.273 & 4.254 & 4.238 & & & & & & & \\
\hline 11 & 4.370 & 4.338 & 4.311 & 4.286 & 4.266 & 4.248 & & & & & & & & \\
\hline 12 & 4.358 & 4.328 & 4.302 & 4.279 & 4.259 & & & & & & & & & \\
\hline
\end{tabular}


Tab. 4.Ratios of average specific heat $\bar{c}_{s} / \bar{c}_{s 1}=f\left(T_{s 1}, z\right)$

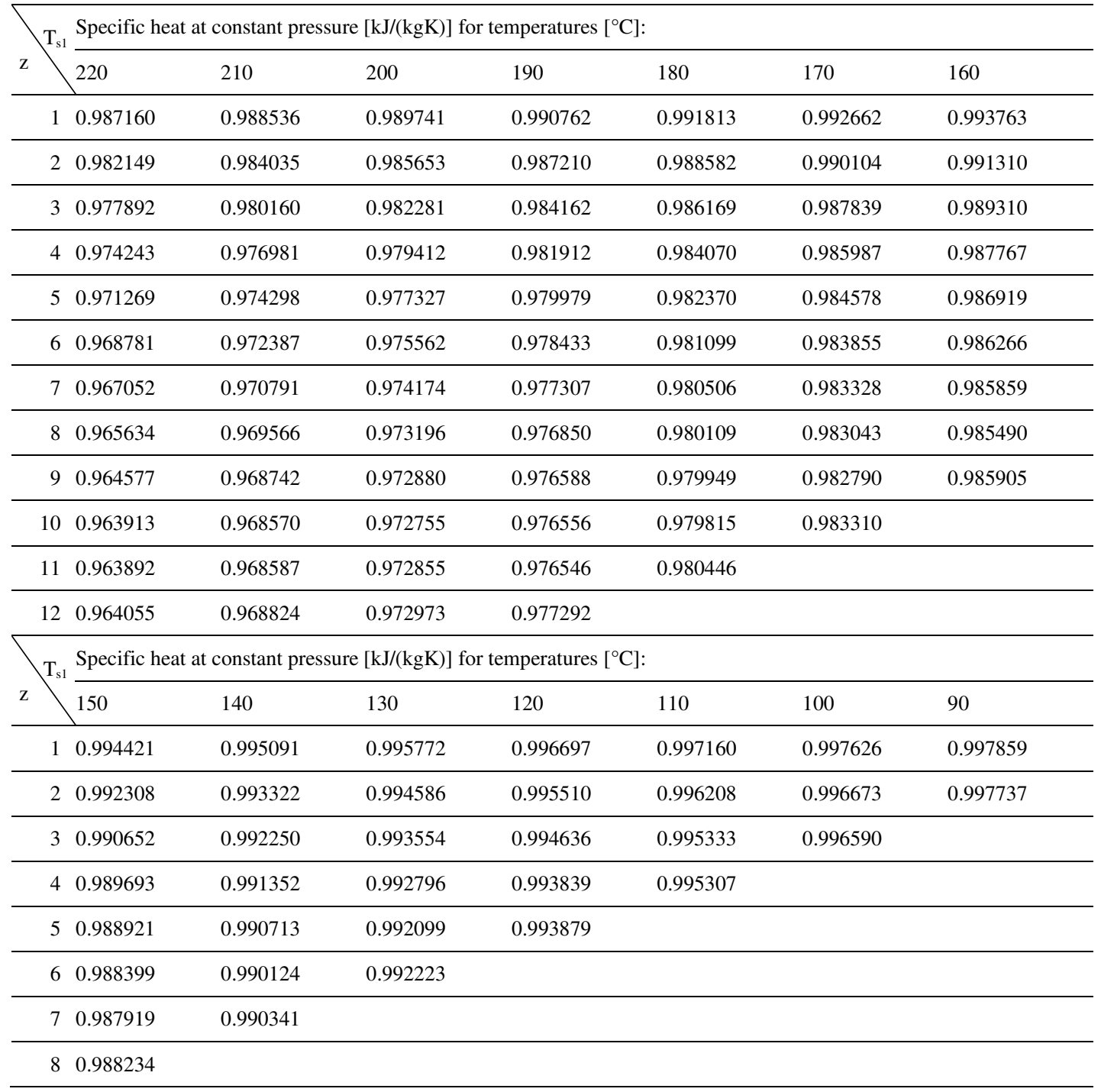

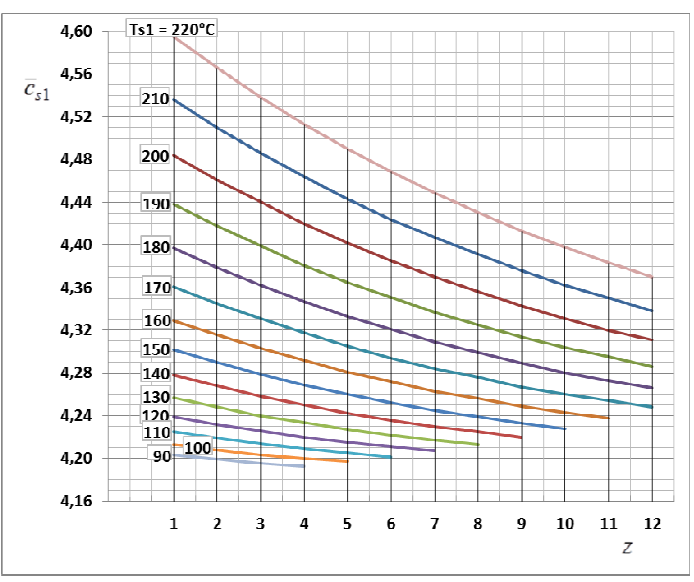

Fig. 5. Average specific heat $\bar{c}_{s 1}=f\left(T_{s 1}, z\right)$

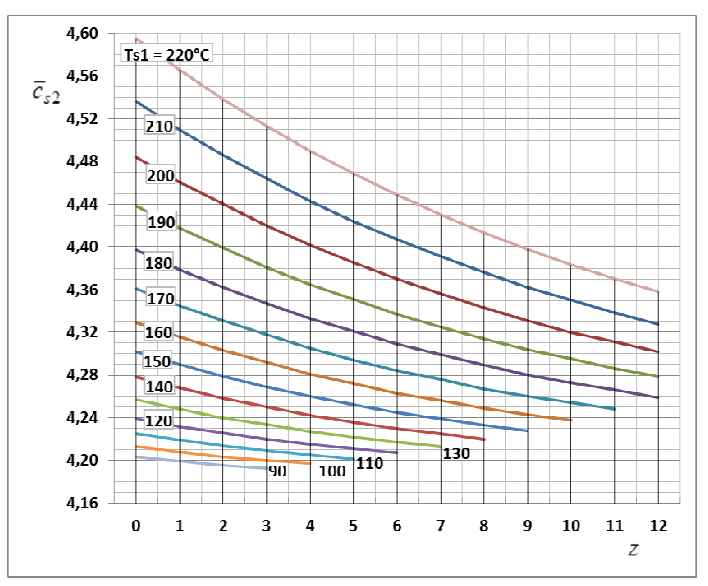

Fig. 6. Average specific heat $\bar{c}_{s 2}=f\left(T_{s 1}, z\right)$ 
Tab. 5.Ratios of average specific heat $\bar{c}_{s} / \bar{c}_{s 2}=f\left(T_{s 1}, z\right)$

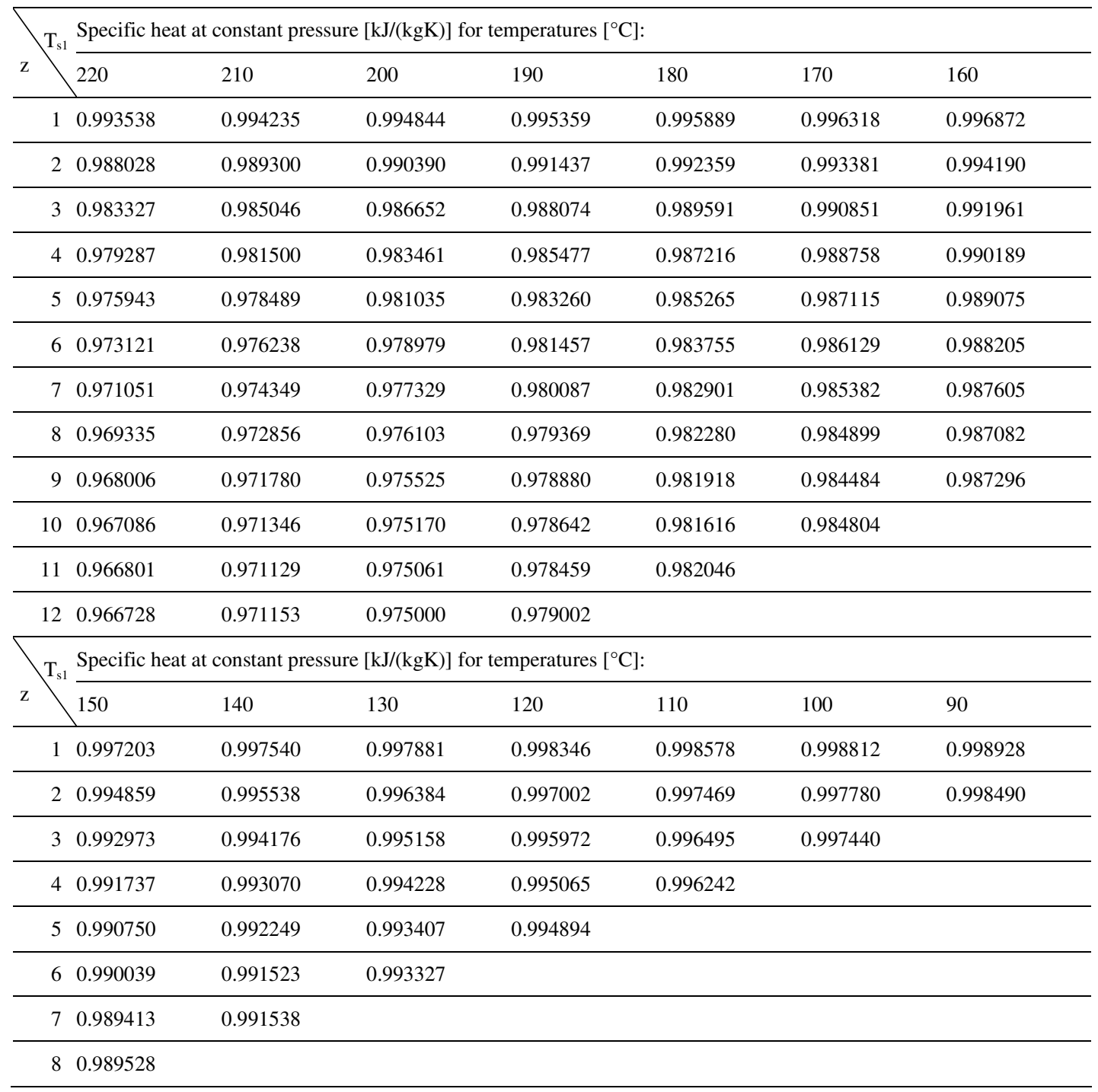

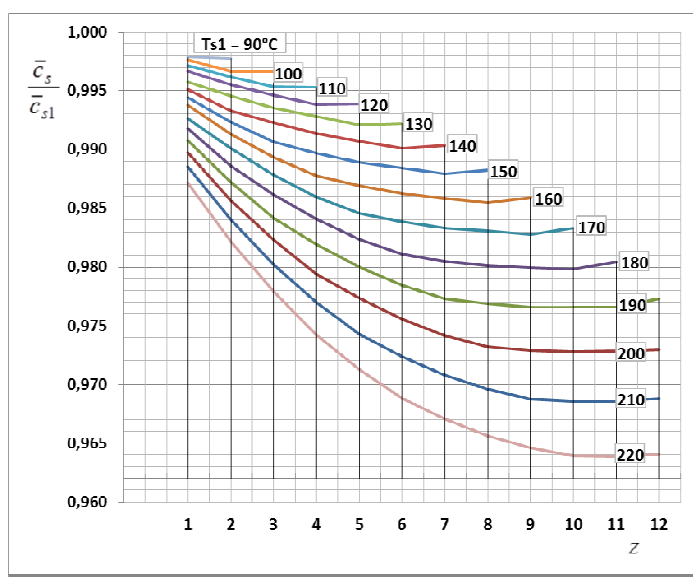

Fig. 7. Ratio of average specific heat $\bar{c}_{s} / \bar{c}_{s 1}=f\left(T_{s 1}, z\right)$

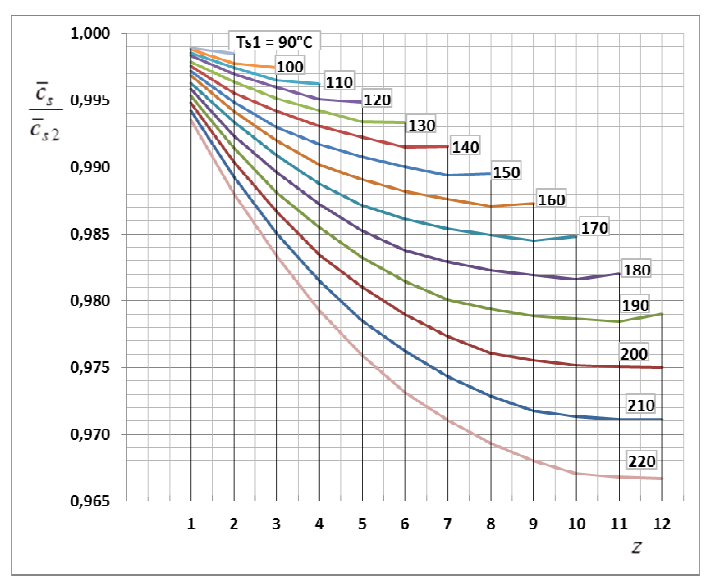

Fig. 8. Ratio of average specific heat $\bar{c}_{s} / \bar{c}_{s 2}=f\left(T_{s 1}, z\right)$ 
Tab. 6. Coefficient $\beta=f\left(T_{s 1}, z\right)$

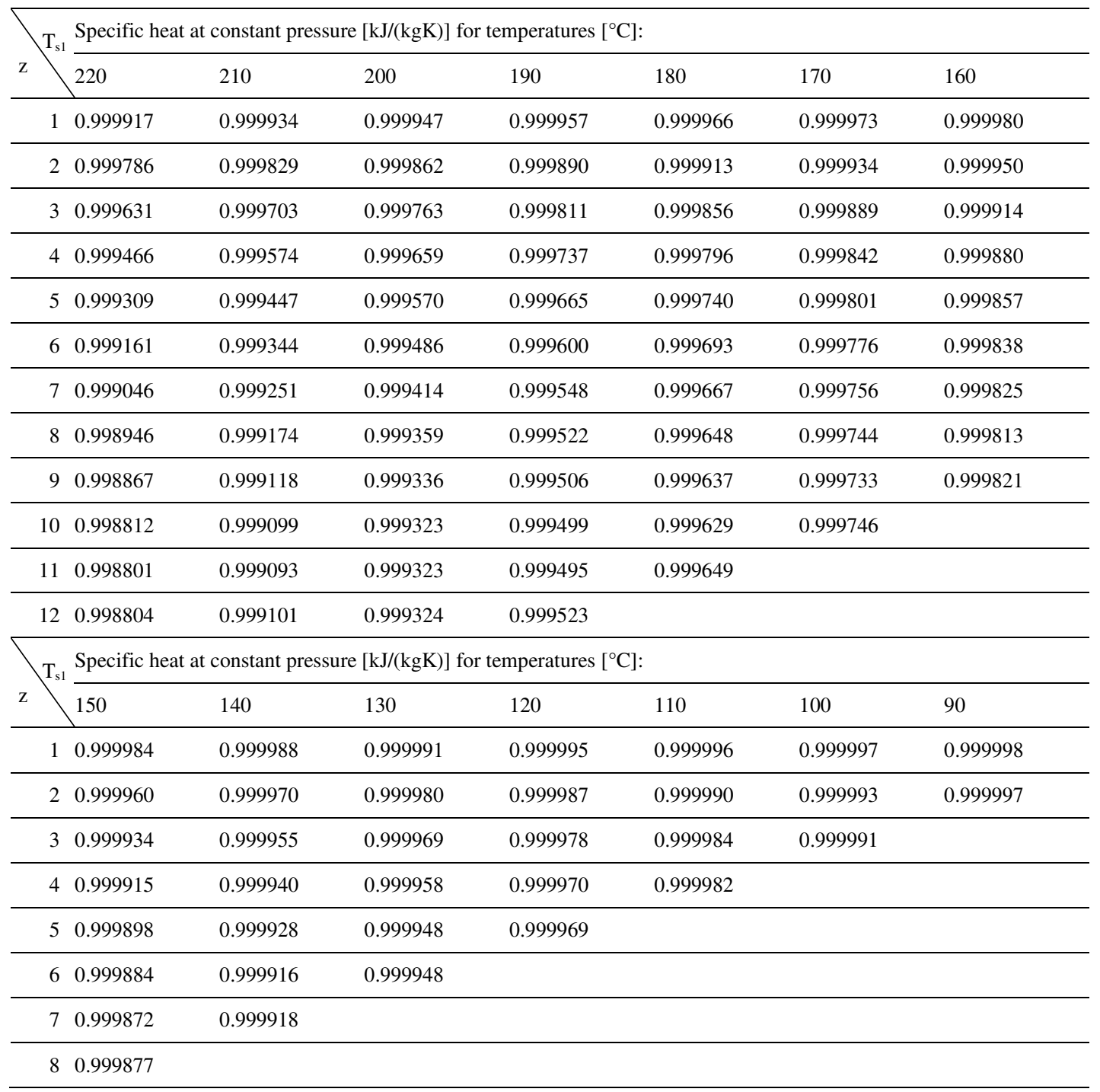

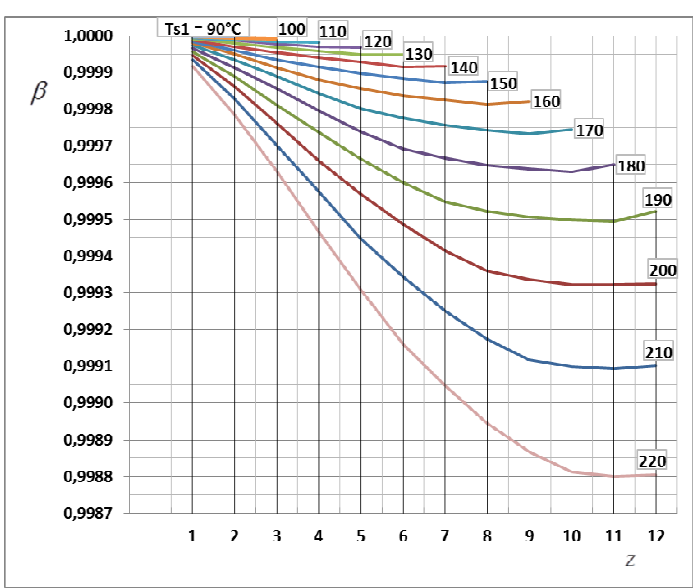

Fig. 9. Coefficient $\beta=f\left(T_{s 1}, z\right)$
And basing on formula (9a), the temperature of recirculating water at the evaporator outlet can be calculated according to

$$
T_{s 2}^{z}=T_{s 1}-(1+z) \cdot \Delta T_{s}^{z}
$$

The heat flux delivered to evaporating medium, taking into account (10) and

$$
\beta=f\left(T_{s 1}, z\right) \cong 1,
$$

takes the following form:

$$
\dot{Q}_{s}=(1+z) \dot{m}_{s} \bar{c}_{s}\left(T_{s 1}^{z}-T_{s 2}^{z}\right)=(1+z) \dot{m}_{s} \bar{c}_{s} \Delta T_{s}^{z}
$$




\section{CONCLUSIONS}

Utilisation of an evaporator with enhanced flow rate of heat carrier, achieved with heat carrier recirculation leads to lower temperature of heat carrier at evaporator inlet due to mixing of streams with different temperatures. The temperature at evaporator outlet, due to heat transfer, is also lower. However, the mass flow rate of heat carrier increases to $\dot{m}_{s}(1+z)$. The enhancement of flow rate can be measured with recirculation coefficient $z$. An ORC power plant with enhanced flow through evaporator, with proper selection of operational characteristics and working fluid, can reach near critical conditions. It also enhances the flow rate of working fluid due to lower latent heat of vaporization at higher temperatures (and reaches 0 at critical point).

The analysis of $\bar{c}_{s}, \bar{c}_{s 1}$ and $\bar{c}_{s 2}$ shows that the average specific heats do not have significant impact on $T_{s 1}^{z}, T_{s 2}^{z}$ and $\Delta T_{s}^{z}$

The development of computational model and example of its use are presented in the research project [12]. This computational model was used in [13].

\section{Acknowledgements}

This paper is a part of the research project NN 513 393436 financed by the Polish National Science Centre.

\section{References}

1. Badr O., Probert S.D., O’Callaghan W.P. (1985) Selecting a working fluid for a Rankine-cycle engine. Applied Energy, Vol. 21, No. 1, pp.1-42.

2. Borsukiewicz-Gozdur A., Nowak W. (2007). Comparative analysis of natural and synthetic refrigerants in application to low temperature ClausiusRankine cycle. Energy, Vol. 32, No. 4, pp. 344-352.

3. DiPippo R. (2008). Geothermal power plants. Principles, applications, case studies and environmental impact. Elsevier, Amsterdam.

4. Gupta H., Roy S. (2007). Geothermal energy: an alternative resource for the $21^{\text {st }}$ century. Elsevier, Amsterdam

5. Kujawa T., Nowak W. (2018). The range of application of ORC power plant in power stations fed with low and medium thermal sources. E3S Web Conf. Vol. 70, https://doi.org/10.1051/e3sconf/20187001009.

6. Lakew A.A., Bolland O. (2010). Working fluids for lowtemperature heat source. Applied Thermal Engineering, Vol. 30, No. 10, pp. 1262-1268.

7. Mikielewicz D., Mikielewicz J. (2010). A thermodynamic criterior for selection of working fluid for subcritical and supercritical domestic micro CHP. Applied Thermal Engineering, Vol. 30, No. 16, pp. 2357-2362.

8. Mikielewicz J., Mikielewicz D. (2009). Comparitive study selected fluids for use in supercritical Organic Rankine Cycles. Archives of Thermodynamics, Vol. 30, No. 2, pp. 2-15.

9. NISIT. (2010). RefProp 9.0, Standard Reference Database 23, Version 9.0, Reference Fluid Thero- dynamic and Transport Properties. National Institute of Standards and Technology, Gaithersburg, MD, USA.

10. Nowak W., Borsukiewicz-Gozdur A., Klonowicz P., Stachel A., Hanausek P., Klonowicz W. (2010). Preliminary results of testing the prototype system of mini-water plants with ORC fed with water at $100^{\circ} \mathrm{C}$. Geological Review, Vol. 58, No. 7, pp. 622-625.

11. Nowak W., Borsukiewicz-Gozdur A., Stachel A. A., Klonowicz W., Hanausek P., Klonowicz P., Magiera R. (2010). Pierwsza demonstracyjna siłownia małej mocy w Polsce. Geological Review, Vol. 58, No. 7.

12. Nowak et al. (2012). Analiza i ocena wptywu bliskokrytycznych warunków wrzenia czynników obiegowych na poprawe efektywności pracy elektrowni geotermalnej. Raport końcowy projektu badawczego NN 513393436 finansowanego przez Narodowe Centrum Nauki.

13. Nowak W., Wiśniewski S. (2011). A concept to fuel the ORC power plant by heat sources of different temperatures, Thermodynamics in Science and Technology Proceedings of the 1st International Congress on Thermodynamics, Part 2, L. Bogusławski (Ed.) Poznań, Poland, pp. 877-886.

14. Saleh B., Koglbauer G., Wendland M., Fischer J. (2007). Working fluids for low-temperature Organic Rankine cycles. Energy, Vol. 32, No. 7, pp. 1210-1221.

15. Weisser P., Skotnicki P. (2010). Perspectives of using ORC systems for low temperature waste energy. Publisher: Instytut Śląski.

16. Wiśniewski S., Nowak W. (2012) ORC power plant effectiveness as affected by the cycle working fluid evaporation at narrow subcritical conditions. 3rd International Conference on Contemporary Problems of Thermal Engineering, CD, CPOTE 2012, Gliwice, Poland.

\section{Biographical notes}

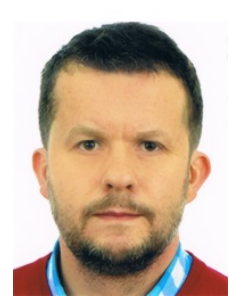

Tomasz Kujawa received his M.Sc. degree in Mechanical Engineering (specialization: Thermal Energy Systems) from Mechanical Faculty and next Ph.D (with honors) degree in Construction from Faculty of Civil Engineering and Architecture, Technical University of Szczecin, in 1993 and 2003, respectively. Since 1993 he has been a researcher in Department of Heat Engineering, Faculty of Mechanical Engineering and Mechatronics, West Pomeranian University of Technology, Szczecin (earlier Technical University of Szczecin). He currently works as a lecturer. His scientific interests focus on problems of obtaining geothermal energy (ground heat exchangers) and ORC installations. He has participated in over 8 national research projects, presenting results of his work at international and national conferences, published more than 80 scientific papers in international and national journals, book chapters, as well as conference proceedings. He is also the co-author of 2 monographs.

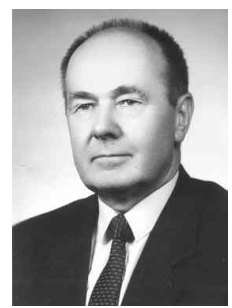

Władysław Nowak received his M.Sc. degree in specialization of Shipbuilding and next Ph.D as well as D.Sc. degree from Gdańsk University of Technology, in 1959, 1965 and 1971, respectively. From 1978 he was as an associated professor, from 1991 to the position of full professor. From 1957 until his retirement, he worked 
continuously at the Faculty of Mechanical Engineering at the Szczecin University of Technology. In his professional life he performed many functions, among others vice-dean and dean of the Faculty of Mechanical Engineering and rector of the Szczecin University of Technology. In the years 1978-2003 he was the head of the Department of Heat Engineering. Scientific and research interests include: renewable energy sources (with ORC), geothermal heat plants, heat management, ventilation and air conditioning, heat exchange and heat exchangers. His specialty is thermal technology, especially heat transfer, has a significant scientific output, including authorship or co-authorship of 5 monographs, 6 didactic scripts, 5 patents, 3 patent applications and over 450 original scientific publications in major national and international scientific journals and conference materials. He promoted 11 doctors, 4 of whom received a postdoctoral degree and 3 are titular professors. He is a reviewer of 17 habilitation dissertations and 27 doctoral dissertations from Poland and abroad. He made 10 opinions for awarding the title of professor and a dozen for the position of full professor. Promoter of over 250 diploma theses. Organizer and co-organizer of national and international symposia and conferences, including: Heat Transfer and Renewable Sources of Energy. From January 1, 2003, Professor Władysław Nowak is retired, still working actively in the field of science and playing an important role in the activity and development of the parent unit, which is the Department of Heat Engineering. 